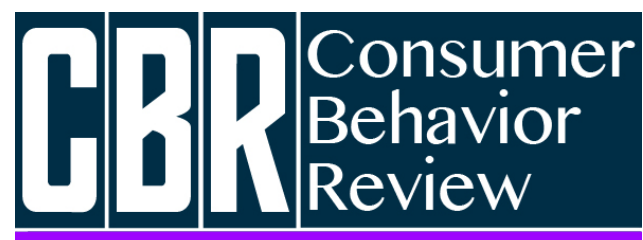

Revista Comportamento do Consumidor
Bornaschella, G., Falcão, R. F., \& Hamza, K. M. (2020). Determinantes da Satisfação e da Recompra na Economia Compartilhada: Um estudo sobre o Airbnb. Consumer Behavior Review, 4(2), 103-117.
ISSN: 2526-7884

Editor: Prof. Dr. Marconi Freitas da Costa Journal's e-mail: cbr@ufpe.br
Evaluation: Double blind review Received: 06 de dezembro de 2019 Accepted: 27 de fevereiro de 2020

\title{
DETERMINANTES DA SATISFAÇÃO E DA RECOMPRA NA ECONOMIA COMPARTILHADA: UM ESTUDO SOBRE O AIRBNB
}

\author{
Determinants of Satisfaction and Repurchase in the Sharing Economy: A study on Airbnb
}

\author{
Giovanna Bornaschella ${ }^{1}$ \\ ORCID: https://orcid.org/0000-0002-6443-4022 \\ E-mail: gibornaschella@gmail.com \\ Roberto Flores Falcão \\ ORCID: https://orcid.org/0000-0002-3153-8650 \\ E-mail: roberto.falcao@unialfa.com.br \\ Kavita Miadaira Hamza ${ }^{1}$ \\ ORCID: https://orcid.org/0000-0002-3586-5587 \\ E-mail: kavita@usp.br
}

\begin{abstract}
${ }^{1}$ Faculdade de Economia, Administração e Contabilidade, Universidade de São Paulo, São Paulo, Brasil ${ }^{2}$ Mestrado Profissional de Administração, Centro Universitário Alves Faria, São Paulo, Brasil
\end{abstract}

\section{Resumo}

Compartilhar é algo trivial na sociedade, mas a internet modificou e facilitou os modos de se compartilhar, ampliando sua escala e gerando a Economia Compartilhada. 0 objetivo deste estudo é aplicar o modelo de Möhlmann (2015) sobre fatores de influência na escolha de consumo colaborativo, analisando-se os usuários do Airbnb. Foram coletados 108 questionários com base em 11 hipóteses, cujos dados foram submetidos a um modelo de equações estruturais, usandose o SmartPLS 2.0. Como principais achados,

\begin{abstract}
Sharing is trivial in society, but the internet has changed and facilitated ways of sharing, expanding its scale and generating the Sharing Economy. The aim of this study is to apply the model of Möhlmann (2015) on factors influencing choice of collaborative consumption by analyzing Airbnb users. We collected 108 questionnaires based on 11 hypotheses. The data were then submitted to a structural equation model using SmartPLS 2.0. As main findings, four relevant factors were identified to explain satisfaction:
\end{abstract}


identificaram-se quatro fatores relevantes para a satisfação com a escolha: comunitarismo, afinidade com tendências, confiança e qualidade do serviço. Já para a probabilidade de recompra do serviço, foram identificados os fatores: confiança, capacidade da internet e satisfação com o serviço. Os resultados contribuem para a discussão acerca do comportamento do consumidor na economia compartilhada e agrega conhecimento ao setor estudado. Também fornece insights para o emergente modelo econômico e empresas que oferecem tais serviços.

Palavras-chave: Economia Compartilhada, Satisfação, Airbnb. communitarianism, affinity with tendencies, trust and quality of service. For the probability of repurchasing the service, the following factors were identified: trust, internet capacity and satisfaction with the service. The results contribute to the discussion about consumer behavior in the shared economy and aggregate knowledge to the sector studied. It also provides insights for the emerging economic model and companies offering such services. Keywords: Sharing Economy, Satisfaction, Airbnb.

\section{INTRODUÇÃO}

Compartilhar pode ser definido como o ato ou processo de distribuir o que é próprio de uma pessoa para o uso de outros, e/ou o ato ou processo de receber algo de outros para o uso particular (Belk, 2007). Embora o ato de compartilhar - com familiares, amigos ou desconhecidos - ocorra há anos na sociedade, o advento da internet modificou e facilitou os modos para se compartilhar, ampliando a escala dessa ação (Belk, 2010). Adicionalmente, o aquecimento global, o aumento dos preços das matérias-primas, a elevação dos níveis de poluição e outras crises de recursos, geraram oportunidades - e talvez a necessidade - para um consumo colaborativo (Belk, 2010). Para Albinsson e Perera (2012) esses fatores levaram à uma maior conscientização dos consumidores acerca das consequências sociais negativas de um consumo exagerado que os levou a procurar por formas alternativas de consumo. Nesse cenário, viabiliza-se o compartilhamento como modelo de negócio, culminando no que hoje é chamado de Economia Compartilhada, um fenômeno mundial, em rápida ascensão, que transforma o conceito de valor para o consumidor, impactando sua renda e tempo, o meio ambiente e a economia como um todo (Botsman \& Rogers, 2010).

Já em 2011, a Time Magazine nomeou a Economia Compartilhada como "uma das 10 ideais que irão mudar o mundo" (Walsh, 2011). A relevância do tema também foi demonstrada por uma pesquisa da Nielsen (2014) realizada mundialmente. Nela, 68\% dos entrevistados estão dispostos a compartilhar seus bens, enquanto $66 \%$ dizem estar propensos a usar bens ou serviços de outros em um modelo de compartilhamento. Para o Brasil, a intenção de participar de alguma iniciativa de consumo compartilhado seria de 78\%. Considerando somente os setores nos quais está mais presente (automotivo, varejo e bens de consumo, hoteleiro e de mídia e entretenimento), a Economia Compartilhada gera uma receita anual de 15 bilhões de dólares, segundo estimativa da consultoria PriceWaterhouseCoopers. A consultoria também projeta que, em 2025, para os mesmos setores, esse valor alcançará a casa dos 335 bilhões de dólares. No que se refere à riqueza gerada exclusivamente para os usuários da Economia Compartilhada, a Forbes, em 2013, estimou um valor em torno de 3,5 bilhões de dólares, com crescimento próximo a 25\% a cada ano (Geron, 2013). Em complemento à expressividade dos números, Botsman e Rogers (2010) sugerem que o consumo colaborativo pode ser tão importante quanto a Revolução Industrial no que tange ao modo como a sociedade pensa sobre a propriedade.

Apesar da crescente importância do consumo colaborativo, há uma falta de estudos quantitativos sobre fatores motivacionais que afetam as atitudes e intenções dos consumidores em relação a esse novo negócio (Hamari, Sjöklint \& Ukkonen, 2014), especialmente no contexto brasileiro. 
Um dos estudos que visaram quebrar esse paradigma foi proposto por Möhlmann (2015). Nele, a autora desenvolve um quadro a respeito dos fatores de influência sobre a escolha de uma opção de consumo colaborativo, validando-o em dois estudos quantitativos: com usuários alemães do serviço de compartilhamento de automóveis Car2Go e, do serviço de locação e hospedagem, Airbnb. Ao final de seu trabalho, a autora sugere que pesquisas futuras investiguem casos adicionais em outras indústrias de consumo colaborativo e/ou outros países para fortalecer o emergente modelo econômico com informações. Desse modo, o presente estudo visa aplicar o modelo desenvolvido por Möhlmann (2015) para usuários do serviço de acomodação e hospedagem Airbnb no Brasil. Neste contexto, o presente estudo se propõe a responder às seguintes questões de pesquisa:

Questão de pesquisa 1: Quais são os fatores de influência mais importantes para explicar a satisfação dos usuários do Airbnb com a utilização dessa opção de compartilhamento?

Questão de pesquisa 2: Quais são os fatores de influência mais importantes para explicar a probabilidade dos usuários do Airbnb de escolher novamente essa opção de compartilhamento?

\section{ECONOMIA COMPARTILHADA}

Atualmente, persiste uma dificuldade em definir com precisão a Economia Compartilhada, devido às amplas variações na terminologia existente e ao fato do consumo colaborativo se manifestar em diferentes graus de intercâmbio digital e físico (Hamari, Sjöklint \& Ukkonen, 2014). Sob a ótica de Belk (2014), a Economia Compartilhada refere-se à distribuição ou aquisição de recursos, em troca de uma taxa ou outra compensação, organizados por grupos de indivíduos. Ao incluir outras compensações, o autor permite que a definição englobe também trocas ou negociações que envolvam a concessão e o recebimento de compensações não monetárias. Entretanto, Belk (2014) admite que tal definição não se enquadra tão perfeitamente no cenário atual, pois ao excluir as atividades de compartilhamento sem compensações envolvidas, negligenciam-se as doações e iniciativas como as do CouchSurfing.

Por sua vez, Botsman e Rogers (2010) conceituam a Economia Compartilhada, como um conjunto de práticas comerciais que possibilitam o acesso a bens e serviços, sem que haja, necessariamente, a aquisição dos mesmos ou troca monetária entre as partes envolvidas. Essas práticas são constituídas por transações como compartilhamento, empréstimo, aluguel, doação, trocas e escambo. Em complemento, para esses autores, a economia do compartilhamento é um fenômeno econômico-tecnológico emergente, alimentado pela evolução das tecnologias da informação e comunicações (TIC), pela crescente conscientização dos consumidores, pela proliferação de comunidades web colaborativas.

Já Schor (2014) criou quatro categorias para enquadrar os diversos tipos de atividades da Economia Compartilhada: recirculação de bens, maior utilização de ativos duráveis, troca de serviços e compartilhamento de ativos produtivos. A primeira refere-se à venda dos mais diversos bens usados em mercados físicos ou virtuais. 0 segundo tipo de plataforma promove o uso mais intensivo de bens duráveis e outros ativos, cuja capacidade não é plenamente atingida por um único proprietário como, por exemplo, quartos não ocupados pelos moradores de uma casa. Comercialmente, pode-se citar empresas como a Zipcar e o Airbnb, por exemplo. Já as iniciativas não monetárias geralmente baseiamse em ações de vizinhança como bibliotecas comunitárias.

A troca de serviços, por sua vez, é uma modalidade na qual serviços são negociados com base no tempo gasto por cada indivíduo ao desenvolver uma atividade para outrem. Exemplos sem fins lucrativos seriam os bancos de tempo - uma ação iniciada na década de 1980, nos Estados Unidos que encontra espaço, atualmente, em comunidades locais. Já intercâmbios de serviços monetizados incluem o Task Rabbit e a Zaarly. Por fim, a quarta categoria consiste em esforços focados no compartilhamento de ativos ou espaços para propiciar a produção em detrimento do consumo. As cooperativas são a forma histórica que esses esforços tomaram. Modernamente, iniciativas relacionadas são espaços de coworking e sites como o Skillshare.com - uma plataforma educacional de livre produção de conteúdo. 
Schor (2014) categoriza ainda as atividades da Economia Compartilhada segundo outras duas perspectivas: o propósito para o qual é orientada e o tipo de fornecedor. Nesse contexto, as plataformas poderiam ser com ou sem fins lucrativos, sendo que, se orientada para gerar lucros, influenciará a forma como o compartilhamento ocorre e a divisão da receita entre intermediador e proprietário. Quanto ao tipo de fornecedor, as plataformas podem ser P2P (peer-to-peer) e B2P (business-to-peer). As entidades P2P ganham dinheiro por comissões sobre câmbio, de modo que o crescimento da receita depende do aumento do número de negócios. Com uma estrutura P2P, os "pares" devem ser capazes de capturar uma maior fração de valor para si. Em contrapartida, as plataformas B2P buscam maximizar a receita por transação, como as empresas tradicionais costumam fazer. Este estudo analisará o Airbnb, uma plataforma com fins lucrativos e que se caracteriza como P2P.

\section{Variáveis de influência do consumidor na economia compartilhada}

Dada a diversidade de plataformas e atividades existentes da Economia Compartilhada, os motivos que levam os indivíduos a participar em tais ações, também são diversos (Schor, 2014). Möhlmann (2015) desenvolveu um framework sobre os atributos determinantes para a escolha de uma opção de compartilhamento e sua satisfação com a mesma. Assim, a autora, após um levantamento da literatura relevante sobre o tema, estrutura seu trabalho segundo onze atributos que são testados em dois estudos quantitativos e validados ao aplicar análises de modelagem de caminho de mínimos quadrados parciais. São pesquisados, no estudo 1, usuários do serviço de compartilhamento de carro Car2Go $(\mathrm{N}=236)$ e, no estudo 2 , os usuários do marketplace de hospedagem Airbnb ( $\mathrm{N}=187)$. De maneira geral, os resultados revelaram que tanto a satisfação como a probabilidade de escolher uma opção de compartilhamento novamente, podem ser explicadas predominantemente por determinantes que atendem ao benefício próprio do indivíduo.

Möhlmann (2015) ressalta a importância de seus resultados para a gestão de negócios voltados para o consumo colaborativo, uma vez que se desenvolve o conhecimento sobre o comportamento dos consumidores e as razões para o uso dos serviços. Poder-se-ia assim gerenciar estrategicamente relacionamentos e desenvolver estratégias mais assertivas e direcionadas a clientes atuais e potenciais. Isto posto, o artigo elaborado por Möhlmann (2015) embasou uma variedade de estudos que contribuíram para o escopo do estudo do comportamento do consumidor na Economia Compartilhada, porem nenhum deles no contexto brasileiro.

0 presente estudo avaliou a relevância de fatores de influência geralmente associados à decisão de compra/recompra no contexto da Economia Compartilhada, como ressaltado anteriormente. Tais variáveis são: comunitarismo ou senso de pertencimento a uma comunidade, economia de custos, impacto ambiental, familiaridade com a plataforma, capacidade de acesso à internet, qualidade do serviço, capacidade do smartphone, afinidade com tendências, confiança e utilidade.

O comunitarismo refere-se à disposição do consumidor em utilizar um serviço com o intuito de pertencer a uma comunidade de indivíduos semelhantes. Botsman e Rogers (2010) justificam a ascensão do comunitarismo como um resultado do atual ambiente social, repleto de opções que facilitam o processo de uma pessoa conectar-se às outras com ideias e atitudes semelhantes. Tal possibilidade de conexão propiciada pelas redes sociais, que promovem encontros online e facilitam os off-line, impactaram a nova geração de millenials que desenvolveram quase uma necessidade de conexão.

Já Albinsson e Perera (2012) analisaram formas de consumo alternativas em eventos de compartilhamento não monetários. Seu estudo indicou que o senso de comunidade é, simultaneamente força motriz e o resultado da participação dos indivíduos em tais eventos. Os participantes praticariam o consumo colaborativo movidos pela ideologia de conectarem-se à comunidade a qual pertencem e ajudarem em sua construção por meio de tais trocas. Adicionalmente, Galbreth et al. (2012) ao estudar o compartilhamento social de bens de informação identificou que, além dos benefícios monetários percebidos, os participantes aspiravam o pertencimento à comunidade de partilha estudada. 
Dado o crescimento e o impacto do consumo colaborativo no setor de viagens e turismo, Tussyadiah (2015) buscou estudar as motivações e barreiras para norte-americanos utilizarem serviços de hospedagem peer-to-peer com finalidade lucrativa. Após a análise dos dados, Tussyadiah (2015) concluiu que a interação social era o fator com maior influência para a escolha. Considerando a importância do senso de comunidade para o consumo colaborativo, destacada pelos estudos anteriormente expostos, foram formuladas as duas primeiras hipóteses deste estudo:

H1a: O comunitarismo possui impacto positivo na satisfação com a escolha de uma oferta de economia compartilhada.

H1b: O comunitarismo possui impacto positivo na probabilidade de se utilizar novamente uma opção de economia compartilhada.

A economia de custos é uma variável atrelada ao benefício econômico desfrutado pelo indivíduo, gerado pelo menor desembolso frente a uma alternativa de consumo. Uma pesquisa que endossa a importância da variável é encontrada em Mont (2004) que analisou por meio de entrevistas em profundidade o comportamento de stakeholders em negócios colaborativos. Ao final, a autora conclui que a satisfação dos clientes de compartilhamento de automóveis é positivamente influenciada pela redução em seus custos de transporte, tanto para os usuários que utilizam o serviço, quanto para os que disponibilizam seu veículo para aluguel. Já Lamberton e Rose (2012) estudaram sistemas comerciais de compartilhamento no que tange aos seguintes produtos: automóveis, bicicletas e planos de minutos para telefonia celular. Os autores argumentam que os consumidores apresentam interesse na partilha quando seus custos pessoais são minimizados e, os benefícios percebidos na transação, maximizados.

Por outro lado, Bardhi e Eckhardt (2012) estudaram o acesso temporário a automóveis por meio do pagamento pelo uso. Como resultado, os autores identificaram que os aspectos econômicos, ligados a algum tipo de poupança dos recursos, são a maior motivação dos indivíduos. Em linha com os resultados supracitados, Moeller e Wittkowski (2010) conduziram um estudo quantitativo com alemães membros de uma rede de compartilhamento online. Seus resultados demonstraram que o fato de as opções de compartilhamento serem geralmente mais baratas, é o principal determinante no momento da escolha desse tipo de negócio pelos indivíduos. Adicionalmente, Tussyadiah (2015) também identificou os benefícios econômicos como um fator importante para os usuários. Portanto, os estudos anteriores demonstram que os aspectos econômicos impactam positivamente na escolha de uso da economia colaborativa. Assim, as hipóteses levantadas foram:

H2a: A economia de custos possui impacto positivo na satisfação com a escolha de uma oferta de economia compartilhada.

H2b: A economia de custos possui impacto positivo na probabilidade de se utilizar novamente uma opção de economia compartilhada.

0 impacto ambiental se refere à redução de danos ao meio ambiente propiciado pelo envolvimento com opções de compartilhamento. De maneira ampla, pode-se dizer que as formas de consumo compartilhado são mais sustentáveis por representarem um contraponto em relação aos níveis crescentes de consumo global (Albinsson \& Perera, 2012). Botsman e Rogers (2010) evidenciaram que os usuários de serviços de compartilhamento de automóveis reduzem cerca de $50 \%$ das emissões de gás carbônico per capita. Mont (2004) também mostra que com o consumo colaborativo a superprodução é minimizada, o uso de recursos decresce e o desperdício é evitado.

Albinsson, Wolf e Kopf (2010) identificaram uma tendência à rejeição do consumismo e ao favorecimento de práticas de consumo mais sustentáveis. 0 autor concluiu que possuir bens passa a ter conotações cada vez mais negativas, por seu impacto danoso ao meio ambiente. Isso converge com o trabalho de Ozanne e Ballantine (2010), que após pesquisar o comportamento de membros de uma locadora de brinquedos, verificou uma tendência dos indivíduos de substituir a posse pela partilha por esta última ter menor impacto negativo sobre o meio ambiente. Hamari et al. (2013) buscando investigar os estímulos para o engajamento no consumo colaborativo em sites voltados para a partilha 
de bens, identificaram que as pessoas são atraídas pela sustentabilidade, principalmente quando elas já valorizam um consumo mais responsável. Diversos estudos apontam para uma preocupação com o impacto ambiental por parte dos consumidores e suas preferências de consumo daí derivadas. Neste sentido, foram formuladas as seguintes hipóteses:

H3a: O impacto ambiental possui impacto positivo na satisfação com a escolha de uma oferta de economia compartilhada.

H3b: O impacto ambiental possui impacto positivo na probabilidade de se utilizar novamente uma opção de economia compartilhada.

A familiaridade refere-se à influência que o uso ou conhecimento prévio do serviço tem no comportamento do indivíduo. Moeller e Wittkowski (2010) identificaram que uma possível barreira ao uso de serviços de compartilhamento de automóvel pela primeira vez era a falta de experiências anteriores dos indivíduos. Henning-Thurau et al. (2007) complementam a importância da familiaridade ao estudar o comportamento de consumidores de serviços de compartilhamento de filmes. Os autores comentam que, não ter a habilidade para usar os serviços, configura-se em um impeditivo, ao menos, temporário, para experimentá-los. Por conseguinte, uma maior familiaridade com os produtos da economia compartilhada auxiliaria na adoção de bens e serviços ao minimizar seus custos de mudança e, assim, seria um fator de influência na satisfação e usos futuros da opção de compartilhamento (Möhlmann, 2015). Desse modo, foram formuladas as hipóteses:

H4a: A familiaridade com a plataforma possui impacto positivo na satisfação com a escolha de uma oferta de economia compartilhada.

H4b: A familiaridade com a plataforma possui impacto positivo na probabilidade de se utilizar novamente uma opção de economia compartilhada.

0 próximo fator de influência é a capacidade de acesso à internet do próprio indivíduo, que pode impactar na experiência com o serviço, dado que se tratam de marketplaces. Gardner, Ostrom e Walker (1990) teorizavam que a capacidade de comunicação era o fator determinante para o sucesso de uma transação entre duas partes de qualquer acordo de colaboração. Recentemente, Belk (2010) atribui à internet a ascensão expressiva dessa ação, propiciando seu crescimento até que se tornasse um modelo econômico como é hoje. Botsman e Rogers (2010) também consideram a economia do compartilhamento como um fenômeno econômico-tecnológico que foi fomentado pela evolução das tecnologias da informação e pela proliferação de comunidades web colaborativas. Em decorrência disso, a capacidade de acesso a internet pode ser um fator de motivação ou mesmo desmotivação para as trocas, sendo uma variável relevante a ser considerada (Möhlmann, 2015), levando as seguintes hipóteses:

H5a: A capacidade de acesso à internet possui impacto positivo na satisfação com a escolha de uma oferta de economia compartilhada.

H5b: A capacidade de acesso à internet possui impacto positivo na probabilidade de se utilizar novamente uma opção de economia compartilhada.

Outro fator considerado é a capacidade do smartphone. A possibilidade oferecida pelos smartphones, de reunir em um único aparelho aplicativos de diversas funcionalidades, proporcionando acesso mais fácil e rápido a serviços. Nos estudos de Zhao (2010) e Botsman e Rogers (2010), os smartphones foram citados como um fator que facilitou o acesso ao produto e, por isso, acabou influenciando sua escolha. No contexto da economia compartilhada, o smartphone é uma importante ferramenta para o consumidor - crucial em alguns casos, pois permite aos usuários verificarem a disponibilidade e a localização de carros de maneira imediata, bem como reservá-los e oferecer uma rota opcional para chegar até eles. Ademais, uma maior capacidade do smartphone leva a uma maior satisfação ao se optar pelo consumo colaborativo, levando a formulação das seguintes hipóteses: 
H6a: A capacidade do smartphone possui impacto positivo na satisfação com a escolha de uma oferta de economia compartilhada.

H6b: A capacidade do smartphone possui impacto positivo na probabilidade de se utilizar novamente uma opção de economia compartilhada.

A próxima variável estudada diz respeito à qualidade do serviço prestado. A qualidade de um serviço está intrinsecamente relacionada ao cliente que o consome, a suas expectativas prévias e ao nível de atendimento à tais expectativas proporcionadas pela experiência durante o serviço. Desse modo, um serviço é classificado como de qualidade pelo consumidor quando a qualidade experimentada alcança ou ultrapassa suas expectativas. Por conseguinte, a qualidade de um serviço, eleva a satisfação do consumidor (Hoffman \& Bateson, 2003). É uma opinião estabelecida em pesquisas sobre comportamento de consumidores em serviços que a qualidade percebida é um importante antecedente para a satisfação e para a intenção de utilizar o serviço novamente (Fornell et al.,1996). Assim, foram formuladas as hipóteses:

H7a: A qualidade do serviço possui impacto positivo na satisfação com a escolha de uma oferta de economia compartilhada.

H7b: A qualidade do serviço possui impacto positivo na probabilidade de se utilizar novamente uma opção de economia compartilhada.

A afinidade com tendências indica se o indivíduo é predisposto a utilizar um serviço por puro modismo. Quando diante de um novo produto, o comportamento do consumidor pode ser classificado em 5 grupos: inovadores, adotantes iniciais, maioria inicial, maioria tardia e retardatários. Os dois primeiros perfis são os que apresentam maior predisposição a aceitar novas tendências e, por isso, são considerados chave na introdução de um produto no mercado, pois são os primeiros usuários/consumidores do produto (Rogers, 1965). 0 estudo de Moeller e Wittkowski (2010) também apontou que os indivíduos que almejavam consumir produtos modernos apresentaram uma inclinação a preferir compartilhar ao invés de comprar, indicando que uma maior afinidade com as tendências futuras teria impactos positivos no uso, satisfação e reuso do serviço. Ademais, Schor (2014) ressalta que alguns participantes aderem a modelos de economia compartilhada simplesmente por modismo, pelo destaque dado nas mídias ou pela atração que sentem por novidades ou tecnologias. Portanto, as seguintes hipóteses foram levantadas:

H8a: Afinidade com tendências possui impacto positivo na satisfação com a escolha de uma oferta de economia compartilhada.

H8b: Afinidade com tendências possui impacto positivo na probabilidade de se utilizar novamente uma opção de economia compartilhada.

A confiança é outro determinante considerado para influenciar a satisfação e a recompra de uma opção de economia compartilhada. A confiança e a reciprocidade são os pontos centrais que explicam porque indivíduos - desconhecidos ou não - tendem a cooperar uns com os outros (Cox et al., 2009). Hofmann et al. (2017), estudando serviços de empréstimos de automóveis, identificaram que a confiança é um fator determinante para a adoção de uma opção de compartilhamento quando esta é entre pessoas, sem a intermediação de uma empresa. Kim et al. (2015) também objetivaram estudar o fator da confiança especificamente de usuários da empresa Airbnb, identificando que a confiança seria um fator determinante para a escolha do Airbnb. Desse modo, tem-se as hipóteses:

H9a: A confiança possui impacto positivo na satisfação com a escolha de uma oferta de economia compartilhada.

H9b: A confiança possui impacto positivo na probabilidade de se utilizar novamente uma opção de economia compartilhada. 
Por fim, aborda-se a o efeito do fator utilidade. No âmbito das ciências econômicas, é sabido que os indivíduos têm a tendência de tomar decisões e moldar seus hábitos e atitudes, visando seu interesse próprio e maximizando sua utilidade (Hardin, 1968). Henning-Thurau et al. (2007), em seu estudo sobre compartilhamento de arquivos de vídeo, identificaram que a maximização da utilidade foi um fator significativo para a utilização desse tipo de serviço. Complementarmente, Lamberton e Rose (2012) encontraram que o grau de substituição entre opções de compartilhamento de automóveis era impactado pela busca do usuário para maximizar sua utilidade. Assim, as seguintes hipóteses foram formuladas:

H10a: A utilidade possui impacto positivo na satisfação com a escolha de uma oferta de economia compartilhada.

H10b: A utilidade possui impacto positivo na probabilidade de se utilizar novamente uma opção de economia compartilhada.

A partir das dimensões propostas, os dez fatores de influência enunciados devem testados de modo a averiguar seu efeito sobre a variável endógena: satisfação com uma opção de compartilhamento. Segundo Zeithaml, Bitner e Gremler (2014), a satisfação do usuário em relação a um produto ou serviço é influenciada significativamente pela avaliação que o consumidor faz de suas características. Em complemento, Kotler e Keller (2012) definem a satisfação do consumidor como o sentimento de prazer ou decepção resultante da comparação do desempenho percebido pelo produto com as expectativas pré-estabelecidas pelo cliente. Assim, se o desempenho for inferior às expectativas, o cliente ficará insatisfeito, e, se corresponder às expectativas, o cliente ficará satisfeito.

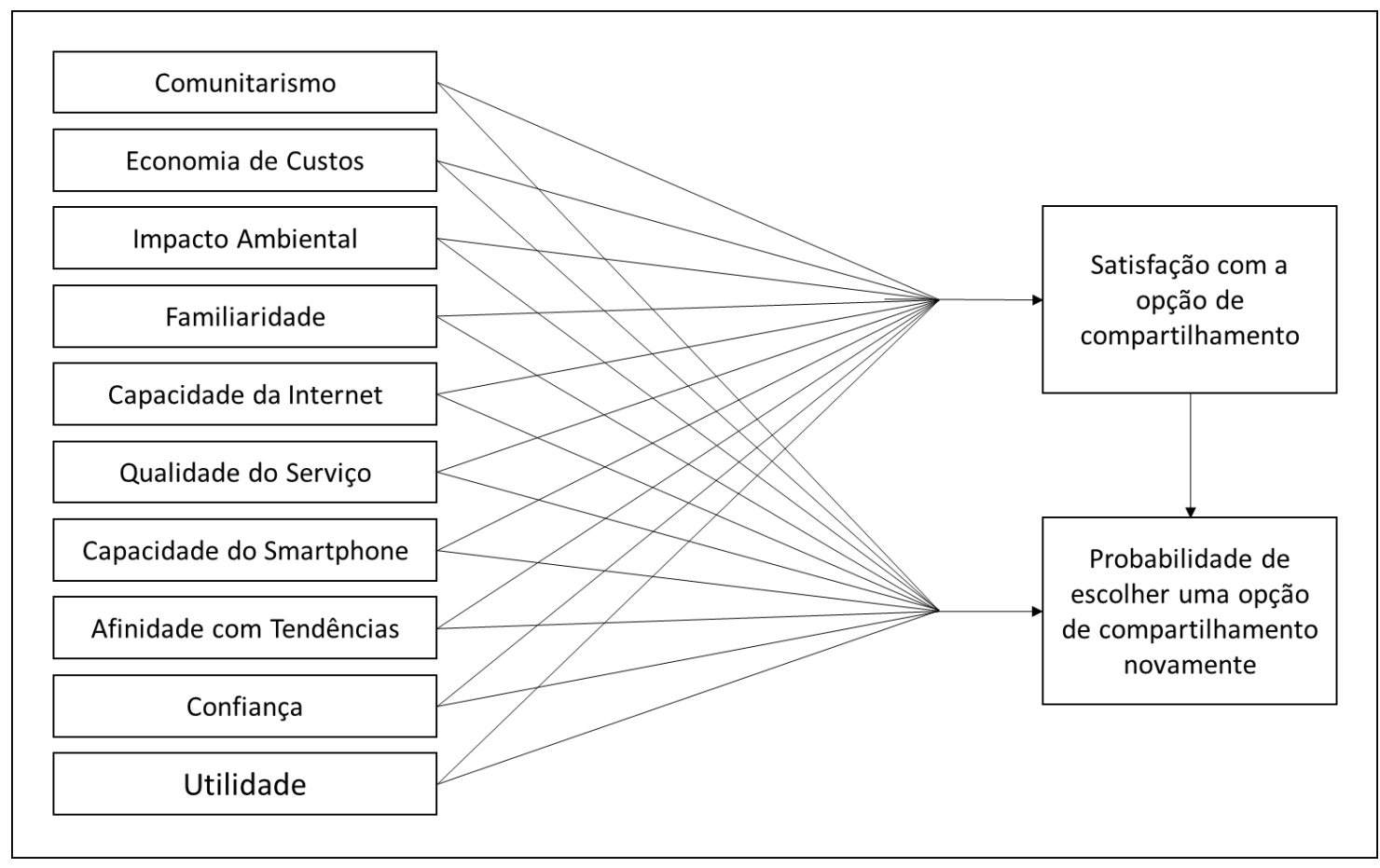

Fonte: Elaborado pelos autores, adaptado de Möhlmann (2015)

Figura 1: Principais influenciadores da satisfação e da probabilidade de se usar novamente uma opção de economia compartilhada

Desse modo, para a outra variável endógena - probabilidade de usar uma opção de compartilhamento em detrimento de uma não compartilhada - além de ser testada quanto aos dez atributos descritos, se acrescentará a influência da variável de satisfação. Isso é verificado baseando-se na opinião estabelecida em pesquisa de marketing e comportamento do consumidor de que a 
satisfação com um serviço pode levar ao reuso (ou recompra) desse serviço específico ou similares no futuro (Fornell et al., 1996; Boenigk \& Helmig, 2013). Formulou-se, assim, a última hipótese:

H11: A satisfação com uma opção de compartilhamento possui impacto positivo na probabilidade de usar uma opção de compartilhamento novamente.

\section{PROCEDIMENTO METODOLÓGICO}

Conforme citado, o objetivo deste estudo é aplicar o modelo desenvolvido por Möhlmann (2015), analisando-se o comportamento dos consumidores na Economia Compartilhada. Para tanto, escolheu-se estudar um caso de serviço colaborativo baseado em plataforma marketplace: locação e hospedagem da empresa Airbnb, que já havia sido analisado no estudo de Möhlmann (2015), porém em outro contexto cultural. Neste estudo, buscou-se comparar os resultados obtidos com os resultados do estudo de Möhlmann (2015), razão pela qual foi escolhida a mesma plataforma de compartilhamento. Por sua vez, as hipóteses decorrentes de tais objetivos foram enunciadas individualmente em seções anteriores. Após a formulação das hipóteses, seguiu-se a etapa de elaboração do questionário. Cada um dos atributos testados foi baseado em multivariáveis reflexivas. As variáveis testadas para a determinação de cada um dos atributos avaliados foram traduzidas, pelos autores, a partir do trabalho de Möhlmann (2015). As versões dos itens traduzidos foram comparadas, e as adaptações necessárias foram realizadas. Para cada variável foram fornecidas sete opções de resposta, cujo nível de concordância variava de $1=$ "Discordo Fortemente" até $7=$ "Concordo Fortemente".

Tabela 1

Variáveis aplicadas nos estudos de caso, segundo cada atributo

\begin{tabular}{|c|c|}
\hline Atributos & Questões \\
\hline \multirow{2}{*}{ Comunitarismo } & $\begin{array}{l}\text { O uso do Airbnb permite que eu seja parte de um grupo de pessoas semelhantes a } \\
\text { mim. }\end{array}$ \\
\hline & $\begin{array}{l}\text { O uso do Airbnb permite que eu faça parte de um grupo de pessoas com interesses } \\
\text { similares aos meus. }\end{array}$ \\
\hline \multirow{2}{*}{$\begin{array}{l}\text { Economia de } \\
\text { Custos }\end{array}$} & Pelo preço dado, considero que as ofertas do Airbnb são boas. \\
\hline & Pela qualidade da oferta do Airbnb eu considero os preços bons/justos \\
\hline \multirow[b]{2}{*}{$\begin{array}{l}\text { Impacto no Meio } \\
\text { Ambiente }\end{array}$} & Ao usar o Airbnb, eu reduzo o uso de recursos naturais. \\
\hline & $\begin{array}{l}\text { Com o uso do Airbnb, eu demonstro um comportamento de consumo amigável ao } \\
\text { meio ambiente. }\end{array}$ \\
\hline \multirow{3}{*}{ Familiaridade } & Estou familiarizado com o processo do Airbnb. \\
\hline & Tenho experiências anteriores com o Airbnb. \\
\hline & No geral, estou familiarizado com o funcionamento do Airbnb. \\
\hline \multirow{3}{*}{$\begin{array}{l}\text { Capacidade da } \\
\text { internet }\end{array}$} & A internet é útil para consumir ofertas do Airbnb. \\
\hline & A internet torna mais conveniente o uso do Airbnb. \\
\hline & Usar a internet torna mais produtivo o uso do Airbnb. \\
\hline \multirow{5}{*}{$\begin{array}{l}\text { Qualidade do } \\
\text { Serviço }\end{array}$} & O design da oferta/site do Airbnb é atrativo para mim. \\
\hline & Eu tenho acesso fácil e rápido a ofertas do Airbnb. \\
\hline & O Airbnb torna fácil para mim concluir a transação. \\
\hline & 0 atendimento ao cliente do Airbnb responde às necessidades de seus clientes. \\
\hline & Eu acredito que o Airbnb conhece as necessidades de seus clientes. \\
\hline \multirow{3}{*}{$\begin{array}{l}\text { Capacidade do } \\
\text { smartphone }\end{array}$} & Meu smartphone é útil para consumir as ofertas do Airbnb. \\
\hline & Meu smartphone me permite um uso conveniente do Airbnb. \\
\hline & Usar meu smartphone deixa o uso do Airbnb mais produtivo. \\
\hline \multirow{2}{*}{$\begin{array}{l}\text { Afinidade com } \\
\text { tendências }\end{array}$} & $\begin{array}{l}\text { O consumo colaborativo da oferta do Airbnb me permite acompanhar as últimas } \\
\text { tendências. }\end{array}$ \\
\hline & $\begin{array}{l}\text { Usar o Airbnb mostra que é importante para mim usar os mais novos bens de } \\
\text { consumo. }\end{array}$ \\
\hline \multirow{2}{*}{ Confiança } & Eu acredito que o produto do Airbnb será igual ao mostrado nas ofertas. \\
\hline & Os outros usuários do Airbnb são verdadeiros ao lidar uns com os outros. \\
\hline
\end{tabular}




\begin{tabular}{|c|c|}
\hline & Os outros usuários do Airbnb não se aproveitarão de mim. \\
\hline & $\begin{array}{l}\text { Eu confio que o provedor do Airbnb fornece proteções suficientes para me proteger da } \\
\text { responsabilidade por danos que eu não sou responsável. }\end{array}$ \\
\hline & O Airbnb fornece um ambiente seguro e robusto no qual eu posso usar o serviço. \\
\hline & Em geral, o Airbnb é confiável. \\
\hline \multirow{2}{*}{ Utilidade } & Eu acredito que uma oferta do Airbnb substitui bem um hotel cor \\
\hline & A oferta do Airbnb é tão boa quanto um hotel. \\
\hline \multirow{3}{*}{$\begin{array}{l}\text { Satisfação com a } \\
\text { opção de } \\
\text { compartilhamento }\end{array}$} & No geral, estou satisfeito com o Airbnb. \\
\hline & O último uso do Airbnb cumpriu minhas expectativas. \\
\hline & $\begin{array}{l}\text { O Airbnb representa a versão ideal de uma opção de compartilhamento de } \\
\text { hospedagem. }\end{array}$ \\
\hline \multirow{3}{*}{$\begin{array}{l}\text { Probabilidade de } \\
\text { escolher a opção } \\
\text { de } \\
\text { compartilhamento } \\
\text { novamente }\end{array}$} & $\begin{array}{l}\text { Provavelmente vou escolher o Airbnb ou uma opção de compartilhamento semelhante } \\
\text { na próxima vez. }\end{array}$ \\
\hline & $\begin{array}{l}\text { No futuro, se eu precisar de uma hospedagem/produto, preferiria uma opção de } \\
\text { compartilhamento como o Airbnb. }\end{array}$ \\
\hline & $\begin{array}{l}\text { No futuro, é provável que escolha uma opção de compartilhamento como o Airbnb em } \\
\text { vez de um hotel/produto. }\end{array}$ \\
\hline
\end{tabular}

Fonte: Elaborada pelos autores.

Além das variáveis essenciais para a mensuração dos atributos, foram adicionadas perguntas gerais para determinação do perfil do respondente como sexo, faixa etária, frequência e tempo de uso dos serviços. A coleta dos dados ocorreu no segundo semestre de 2017, com a distribuição do questionário online enviado por e-mail e disponibilizado em grupos de usuários do Airbnb em redes sociais, de modo a obter uma amostra diversificada.

Para análise das hipóteses, os dados coletados foram submetidos a um modelo de equações estruturais baseado em ajuste de Mínimos Quadrados Parciais com o uso do software SmartPLS 2.0. 0 objetivo é mensurar - por meio de uma série de análises dos mínimos quadrados - as relações causais entre os fatores de influência da satisfação e da probabilidade de utilizar novamente os serviços de compartilhamento sob estudo.

Por fim, para obter os valores $\mathrm{t}$ a fim de testar a significância estatística dos modelos foi aplicado, também no software SmartPLS 2.0, o procedimento de bootstraping (Henseler et al., 2009). Na sequência, a análise do ajuste do modelo é feita em duas etapas: avaliação dos modelos de mensuração - para testar a confiabilidade e validade das variáveis latentes utilizadas no modelo - e avaliação do modelo estrutural - que fornece métricas sobre a qualidade de ajuste do modelo, indicando seu grau de capacidade preditiva. Os resultados de todos os outputs considerados para a validade do modelo foram satisfatórios.

\section{ANÁLISE DOS RESULTADOS}

A amostra do estudo atingiu um total de 108 respondentes. Analisando descritivamente, a amostra é composta majoritariamente por mulheres $(61 \%)$ e por indivíduos em faixas etárias mais novas - $28 \%$ com idade entre 18 e 25 anos e $37 \%$ com idade entre 26 e 25 anos. Acerca da frequência de utilização do Airbnb nos últimos 12 meses, 60\% dos indivíduos declararam utilizá-lo apenas 1 vez, $32 \%$ de 2 a 5 vezes, e os demais $8 \%$, de 5 a 10 vezes. Quanto ao tempo de utilização, $16 \%$ declararam utilizá-lo entre 2 e 3 meses atrás, 29\% há mais de um ano, 37\% entre 2 a 4 anos atrás. Adicionalmente, tem-se que $13 \%$ dos respondentes além de hóspedes, atuam como ofertantes. Na sequência, analisouse o modelo estrutural que fornece informações sobre a relação entre as variáveis latentes e observadas, de modo a atender aos objetivos propostos. Os resultados completos estão descriminados na tabela 2 ao final da seção.

Primeiramente, cinco fatores de influência não tiveram efeito significativo para nenhuma das variáveis dependentes do modelo. A primeira delas, economia de custos, teve ambas hipóteses H2a e H2b rejeitadas, evidenciando que não impacta na satisfação do indivíduo $(-0,21$ n.s.), nem na probabilidade de utilizar novamente a opção $(-0,20$ n.s.). A utilidade também teve suas hipóteses H10a e H10b rejeitadas. Assim, a utilidade também não influencia na satisfação do indivíduo com sua opção de economia compartilhada $(-0,17$ n.s. $)$ e na probabilidade de utilizar novamente a opção $(-0,30$ n.s. $)$. 0 
Airbnb, desse modo, não é percebido como uma alternativa menos dispendiosa que um hotel. Deve-se considerar que, mesmo se o preço do quarto/imóvel alugado for inferior ao de um hotel, o locatário pode não contar com serviço de quarto, refeições e outros produtos/amenidades que podem estar incluídos nas tarifas de um hotel.

0 fator impacto ambiental também não se mostrou relevante para a satisfação com a escolha da opção de compartilhamento (-0,07 n.s.) e para a probabilidade de se utilizá-la novamente $(0,11$ n.s.). Rejeitam-se as hipóteses H3a e H3b. Isso está alinhado com a opinião de autores que questionam a real sustentabilidade proporcionada por negócios da economia compartilhada, como Schor (2014), por exemplo. Ela ressalta que a sustentabilidade é propagada mais no discurso para atender às recentes discussões sobre a preocupação quanto ao uso de recursos naturais. Como o Airbnb oferece um bem durável por um tempo limitado de posse, há falta de evidências físicas de economia de recursos naturais e, por isso, provavelmente o impacto ambiental positivo não é percebido pelos usuários.

As hipóteses $\mathrm{H} 4 \mathrm{a}$ e $\mathrm{H} 4 \mathrm{~b}$ também foram rejeitadas e, assim, evidenciou-se que a familiaridade não é determinante para a satisfação com a escolha da opção de compartilhamento $(0,01$ n.s.) e para a probabilidade de se utilizá-la novamente ( $-0,03$ n.s.). Possivelmente, a familiaridade com a plataforma não é um fator relevante devido ao pouco tempo de existência do Airbnb no mercado e a característica de utilização do serviço cuja necessidade é ocasional e circunstancial. Com relação a este fator, destaca-se também que a plataforma do Airbnb é amigável, intuitiva e muito similar a outras ferramentas de reserva de hospedagem online. 0 último fator que não apresentou efeito positivo para a satisfação com a escolha da opção de compartilhamento $(0,11$ n.s.) e para a probabilidade de se utilizá-la novamente $(-0,39$ n.s.) foi a capacidade do smartphone. Esta dimensão teve, portanto, as hipóteses H6a e H6b, rejeitadas. Desse modo, é possível concluir que a posse de um smartphone não é essencial para que os indivíduos comprem serviços como o Airbnb.

Para os demais fatores de influência, foram encontrados efeitos significativos para uma ou ambas variáveis dependentes do modelo. 0 fator comunitarismo se mostrou relevante para a satisfação com o uso do Airbnb (0,34***) que permite aceitar a hipótese H1a. Esse resultado vai ao encontro de trabalhos que também avaliaram fatores de satisfação com o uso de serviços do setor de hospedagem (Tussyadiah, 2015; Tussyadiah, 2016). Entretanto, a hipótese H1b é rejeitada, pois a variável comunitarismo ter um efeito insignificante $(-0,17$ n.s.) na probabilidade de se utilizar novamente a opção. Esse resultado é devido, possivelmente, por uma característica do perfil dos usuários - 58\% dos respondentes são millenials - caracterizados pela quase necessidade de conexão com outros pares.

A capacidade da internet foi um fator significante para explicar probabilidade de se utilizar novamente a opção de compartilhamento $\left(0,14^{*}\right)$, aceitando-se a hipótese H5b. Entretanto, rejeita-se a hipótese $\mathrm{H} 5 \mathrm{a}$, pois o determinante não se mostrou relevante para a satisfação $(0,12$ n.s.). Possivelmente, como a internet não é uma característica determinante do produto em si, a satisfação com a experiência de aluguel via Airbnb não é impactada por uma maior ou menor capacidade da internet no momento da escolha. Entretanto, a mesma é importante para utilizar o serviço novamente, isso porque, o Airbnb é uma plataforma online. Assim, a inexistência da internet impossibilitaria a existência do negócio. Esse resultado mostra-se ainda mais significativo, pois atribui-se à internet a responsabilidade por disseminar o ato de compartilhar, tornando-o possível em larga escala (Belk, 2010).

Na sequência, demonstrou-se que a qualidade do serviço é significativa para satisfação com opção de compartilhamento $\left(0,38^{* * *}\right)$. Assim, aceita-se a hipótese H7a. Entretanto, não se mostrou relacionada com a probabilidade de se utilizá-lo novamente (0,05 n.s.), rejeitando a hipótese H7b. Esse resultado é corroborado pela teoria do comportamento de consumidor, na qual a qualidade percebida é um importante antecedente para a satisfação com um produto (Fornell et al.,1996). Já a variável afinidade com tendências mostrou-se significante em relação à satisfação $\left(0,16^{*}\right)$, aceitando-se da hipótese H8a. Porém, não se relaciona com a probabilidade de se utilizar novamente a opção de compartilhamento (0,00 n.s.), rejeitando-se a hipótese H8b. Como o Airbnb foi criado há menos de 10 anos, o negócio ainda é considerado uma novidade por muitos, e é um atrativo para o público jovem. 
Esse fato ganha relevância quando se considera que o principal público consumidor pertence a geração Y ou millenial, mais propensos a testarem novidades (Botsman \& Rogers, 2010).

Por sua vez, a confiança entre os membros da plataforma foi o único fator que se mostrou significante tanto para a satisfação $\left(0,17^{*}\right)$ quanto para a probabilidade de se utilizar novamente a opção de compartilhamento $\left(0,19^{*}\right)$. As hipóteses H9a e H9b são aceitas. Esse resultado é importante, uma vez que o Airbnb envolve transações peer-to-peer, na qual a confiança é um fator chave para o sucesso do negócio. Kim et al (2015), estudando usuários do Airbnb, também identificaram a confiança como determinante para a satisfação. Por fim, a satisfação com a opção de compartilhamento foi o fator mais significante para explicar a probabilidade de utilizar novamente o Airbnb $\left(0,39^{* * *}\right)$. Aceitase, pois, a hipótese H11. Este resultado corrobora que ao atender as expectativas dos indivíduos com uma experiência de serviço, estes tornam a utilizá-lo.

Por fim, o coeficiente R2 para a satisfação com a opção de compartilhamento equivaleu a 0,8 e, para a probabilidade de utilizar novamente, 0,67 . Ambos os valores de R2 são considerados adequados e confirmam a validade do modelo construído.

Tabela 2

Coeficientes estruturais para cada variável, resultado do processamento de dados no software PLS 2.0

\begin{tabular}{|c|c|c|}
\hline \multirow{3}{*}{ Hipótese } & \multicolumn{2}{|c|}{ Amostra $(\mathrm{N}=108)$} \\
\hline & \multicolumn{2}{|c|}{ Usuários do Airbnb } \\
\hline & $\begin{array}{l}\text { Coeficiente } \\
\text { de caminho }\end{array}$ & $\begin{array}{c}\text { Hipótese } \\
\text { aceita }\end{array}$ \\
\hline H1a: Comunitarismo $\rightarrow$ Satisfação & $0,34 * * *$ & Sim \\
\hline H1b: Comunitarismo $\rightarrow$ Probabilidade de escolher & $-0,17$ n.s. & Não \\
\hline H2a: Economia de Custos $\rightarrow$ Satisfação & $-0,21$ n.s. & Não \\
\hline H2b: Economia de Custos $\rightarrow$ Probabilidade de escolher & $-0,20$ n.s. & Não \\
\hline H3a: Impacto no Meio Ambiente $\rightarrow$ Satisfação & $-0,07$ n.s. & Não \\
\hline H3b: Impacto no Meio Ambiente $\rightarrow$ Probabilidade de escolher & 0,11 n.s. & Não \\
\hline H4a: Familiaridade $\rightarrow$ Satisfação & 0,01 n.s. & Não \\
\hline H4b: Familiaridade $\rightarrow$ Probabilidade de escolher & $-0,03$ n.s. & Não \\
\hline H5a: Capacidade da internet $\rightarrow$ Satisfação & 0,12 n.s. & Não \\
\hline H5b: Capacidade da internet $\rightarrow$ Probabilidade de escolher & $0,14 *$ & $\operatorname{Sim}$ \\
\hline H6a: Capacidade do smartphone $\rightarrow$ Satisfação & 0,11 n.s. & Não \\
\hline H6b: Capacidade do smartphone $\rightarrow$ Probabilidade de escolher & $-0,39$ n.s. & Não \\
\hline H7a: Qualidade do Serviço $\rightarrow$ Satisfação & $0,38 * * *$ & Sim \\
\hline H7b: Qualidade do Serviço $\rightarrow$ Probabilidade de escolher & 0,05 n.s. & Não \\
\hline H8a: Afinidade com tendências $\rightarrow$ Satisfação & $0,16 *$ & $\operatorname{Sim}$ \\
\hline H8b: Afinidade com tendências $\rightarrow$ Probabilidade de escolher & 0,00 n.s. & Não \\
\hline H9a: Confiança $\rightarrow$ Satisfação & $0,17 *$ & $\operatorname{Sim}$ \\
\hline H9b: Confiança $\rightarrow$ Probabilidade de escolher & $0,19 *$ & $\operatorname{Sim}$ \\
\hline H10a: Utilidade $\rightarrow$ Satisfação & $-0,17$ n.s. & Não \\
\hline H10b: Utilidade $\rightarrow$ Probabilidade de escolher & $-0,30$ n.s. & Não \\
\hline H11: Satisfação $\rightarrow$ Probabilidade de escolher & $0,39 * * *$ & Sim \\
\hline
\end{tabular}

Fonte: Elaborado pelos autores. Nota 1: Satisfação: Satisfação com a opção de compartilhamento; Probabilidade de escolher: Probabilidade de escolher uma opção de compartilhamento novamente". Nota 2: n.s.: não significativo; ${ }^{*} \mathrm{p}<0,05 ;{ }^{* *} \mathrm{p}<0,01 ;{ }^{* *} \mathrm{p}<0,001$. SmartPLS bootstraping: 5000 iterações 


\section{CONCLUSÃO}

O objetivo do presente trabalho almejava responder a duas questões de pesquisa no que se referia ao comportamento do consumidor na economia compartilhada: quais seriam os fatores de influência mais importantes para explicar a satisfação dos usuários do Airbnb e para explicar a probabilidade de se escolher novamente o Airbnb como alternativa de hospedagem. Especificamente, analisou-se a opinião dos usuários do serviço de locação e hospedagem, Airbnb. Para este, foram identificados quatro fatores relevantes para explicar a satisfação com a escolha: comunitarismo, afinidade com tendências, confiança e qualidade do serviço. Já para a probabilidade de utilizar a opção de compartilhamento novamente, foram identificados os fatores: confiança, capacidade da internet e satisfação com o serviço.

Os resultados obtidos por meio deste estudo contribuem para preencher algumas lacunas de pesquisa e enriquecer a discussão acerca do comportamento do consumidor na economia compartilhada no contexto brasileiro. Ao avaliar os fatores importantes para o usuário do serviço, o trabalho contribuiu para agregar conhecimento ao setor estudado e para o emergente modelo econômico. Em complemento, uma análise focada, como realizada na seção anterior também revela as especificidades do negócio. Desse modo, os resultados deste trabalho oferecem insights importantes para não apenas para empresas do setor de hospitalidade, mas para as organizações que atuam na economia compartilhada. A partir dos achados, há evidências que contribuem para a criação de estratégias no sentido de se conhecer melhor o consumidor e assim, adquirir, reter e fidelizar clientes. Os resultados também podem ser úteis para companhias convencionais que podem usar as informações para compreender melhor a economia compartilhada, com uma operação e comportamento do consumidor distintos, tanto para uma futura expansão da atuação da empresa, como para traçar estratégias para neutralizar ações de concorrentes com negócios colaborativos. Por fim, os resultados podem ser usados por instituições sem fins lucrativos que buscam desenvolver estratégias de marketing social para o uso de serviços de consumo colaborativo.

Assim, os profissionais do marketing podem utilizar tais variáveis identificadas de forma direcionada, adaptando as atividades de mercado de forma a oferecer ao público um serviço com os atributos valorizados pelo consumidor. Uma empresa como o Airbnb - ou similar - pode construir seu serviço de modo que sua proposta de valor comunique e ofereça atributos que ressaltem o senso de pertencimento a um grupo, a novidade do serviço, sua qualidade e a confiabilidade. Desse modo, atende-se às expectativas dos consumidores, gerando satisfação com o uso, o que aumentaria a probabilidade de utilização do serviço novamente no futuro. Além disso, a satisfação é uma variável relevante na decisão de recompra de um bem ou serviço. Esses atributos, além de relevantes para o perfil do caso aqui analisado, também se mostraram importantes em estudos de outros setores e países (Zhao, 2010; Kim et al., 2015; Tussyadiah, 2015; Barnes \& Matsson, 2016; Tussyadiah, 2016).

Por fim, o presente trabalho apresenta limitações e sugestões de desenvolvimento. Primeiramente, a amostra do caso analisado não foi construída de modo a representar a distribuição da população brasileira. Um segundo ponto, é que assim como o artigo no qual se baseia, este estudo tem seu escopo limitado à uma plataforma peer-to-peer com fins lucrativos da economia compartilhada. Outros estudos, poderiam abordar serviços de setores distintos e de outras das classificações propostas por Schor (2014). Ao abordar, por exemplo, negócios sem fins lucrativos, poderia-se averiguar a importância de outros atributos para a satisfação com a opção de compartilhamento. Desse modo, pesquisas futuras podem ser conduzidas para aumentar o conhecimento acerca da economia compartilhada que está impactando o mercado tradicional e o comportamento do consumidor.

\section{Referências}

Albinsson, P. A., \& Perera, B. Y. (2012). Alternative marketplaces in the 21st century: Building community through sharing events. Journal of Consumer Behaviour, 7(2), 253-266.

Albinsson, P. A., Wolf, M., \& Kopf, D. A. (2010). Anti-consumption in East Germany: Consumer resistance to hyperconsumption. Journal of Consumer Behaviour, 7(2), 412-425. 
Bardhi, F., \& Eckhardt, G. M. (2012). Access-Based Consumption: The case of car sharing. Journal of Consumer Research, 39(4), 881-898.

Barnes, S. J., \& Mattson, J. (2016). Understanding current and future issues in collaborative consumption: A four-stage Delphi study. Technological Forecasting and Social Change, 104, 200211.

Belk, R. (2007). Why Not Share Rather Than Own? The ANNALS of the American Academy of Political and Social Science, 611(1), 126-140.

Belk, R. (2010). Sharing. Journal of Consumer Research, 36(5), 715-734.

Belk, R. (2014). You are what you can access: Sharing and collaborative consumption online. Journal of Business Research, 67(8), 1595-1600.

Boenigk, S., \& Helmig, B. (2013). Why Do Donors Donate? Examining the Effects of Organizational Identification and Identity Salience on the Relationships among Satisfaction, Loyalty, and Donation Behavior. Journal of Service Research, 16(4), 533-548.

Botsman, R., \& Rogers, R. (2010). O que é meu é seu: Como o consumo coletivo está mudando o nosso mundo. Porto Alegre: Bookman.

Cox, J. C., Ostrom, E., Walker, J. M., Castillo, A. J., Coleman, E., Holahan, R., \& Steed, B. (2009). Trust in Private and Common Property Experiments. Southern Economic Journal, 75(4), 957-975.

Fornell, C., Johnson, M. D., Anderson, E. W., \& Bryant, B. E. (1996). The American Customer Satisfaction Index: Nature, Purpose, and Findings The American Customer Satisfaction Index. Journal of Marketing, 60(4), 7-18.

Galbreth, M. R. Ghosh, B., \& Shor, M. (2012). Social sharing of information goods: Implications for pricing and profits. Marketing Science, 31(4), 603-620.

Gardner, R., Ostrom, E., \& Walker, J. M. (1990). The nature of commom-pool resource problems. Rationality and society, 2(3), 335-358.

Geron, T. (2013). Airbnb and the unstoppable rise of the share economy. Disponível em: www.forbes.com/sites/tomiogeron/2013/01/23/airbnb-and-the-unstoppable-rise-of-theshare-economy/. Acesso em: 15 Mai. 2017.

Hamari, J., Sjöklint, M., \& Ukkonen, A. (2014). The Sharing Economy: Why People Participate in Collaborative Consumption. Journal of the Association for Information Science and Technology, 67(9), 2047-2059.

Hardin, G. (1968). The tragedy of commons. Science, 162(3859), 1243-1248.

Hennig-Thurau, T., Henning, V., \& Sattler, H. (2007). Consumer File Sharing of Motion Pictures. Journal of Marketing, 71(4), 1-18.

Henseler, J., Ringle, C. M., \& Sinkovics, R. R. (2009). The Use of Partial Least Squares Path Modeling in International Marketing. New challenges to international marketing. Emerald Group Publishing Limited., 277-319.

Hofmann, E., Hartl, B., \& Penz, E. (2017). Power versus trust - what matters more in collaborative consumption? Journal of Services Marketing, 31(6), 589-603.

Kim, J., Yoon, Y., \& Zo, H. (2015). Why people participate in the sharing economy: A social exchange perspective. PACIS 2015 proceedings (Paper 76).

Kotler, P., Keller, K. L. (2012). Administração de Marketing. 14 ed. São Paulo: Pearson Prentice-Hall.

Lamberton, C. P., \& Rose, R. L. (2012). When Is Ours Better Than Mine? A Framework for Understanding and Altering Participation in Commercial Sharing Systems. Journal of Marketing, 76(4), 109-125.

Moeller, S., \& Wittkowski, K. (2010). The burdens of ownership: reasons for preferring renting. Managing Service Quality: An International Journal, 20(2), 176-191.

Möhlmann, M. (2015). Collaborative consumption: determinants of satisfaction and the likelihood of using a sharing economy option again. Journal of Consumer Behaviour, 7(2), 253-266.

Mont, 0. (2004). Institutionalisation of sustainable consumption patterns based on shared use. Ecological Economics, 50(1-2), 135-153.

Nielsen (2014). Is sharing the new buying? Reputation and trust are emerging as new currencies. Disponível em: http://www.nielsen.com/gh/en/insights/reports/2014/is-sharing-the-newbuying.html. Acesso em: 20 Set. 2017. 
Gardner, R., Ostrom, E., \& Walker, J. M. (1990). The nature of common-pool resource problems. Rationality and Society, 2(3), 335-358.

Ozanne, L. K., \& Ballantine, P. W. (2010). Sharing as a form of anti-consumption? An examination of toy library users. Journal of Consumer Behaviour, 9(2), 485-498.

Pricewaterhousecoopers (2017). The sharing economy: consumer intelligence series. 2015. Disponível em: http://www.pwc.com/CISsharing. Acesso em: 19 Abr. 2017.

Schor, J. (2014). Debating the Sharing Economy. A Great Transition Initiative Essay, 1-19.

Rogers, E. (1965). Diffusion of Innovations. 3 ed. Londres: Collier Macmillan Publishers.

Tussyadiah, I. P. (2015). An exploratory study on drivers and deterrents of collaborative consumption in travel. In Information and Communication Technologies in Tourism 2015. (pp. 817-830). Springer.

Tussyadiah, I. P. (2016). Factors of satisfaction and intention to use peer-to-peer accommodation. International Journal of Hospitality Management, 55(October), 70-80.

Walsh, B. (2011). Today's smart choice: Don't own. Share. Disponível em: www.time.com/time/specials/packages/article/0,28804,2059521_2059717_2059710,00.html. Acesso em: 19 Abr. 2017.

Zhao, D. (2010). Carsharing: a sustainable and innovative personal transport solution with great potential and huge opportunities. Frost and Sullivan, January, 28.

Zeithaml, V. A., Bitner, M. J., \& Gremler, D. D. (2014). Marketing de Serviços: A Empresa com Foco no Cliente. 6 ed. São Paulo: AMGH. 